\title{
STRATEGY OF ORGANIZATIONAL-TECHNOLOGICAL SOLUTIONS ON PRODUCTION OF LAMB FOR SALE IN FARMS CONDITIONS
}

\begin{tabular}{|c|c|}
\hline \multicolumn{2}{|c|}{$\begin{array}{c}\text { Ivan Mičićl, Zoran Rajić2 }{ }^{2} \text { Ahmet Halilagić }{ }^{3} \\
\text { *Corresponding author E-mail: divanlav@gmail.com }\end{array}$} \\
\hline A R T I C L E I N F O & A B S T R A C T \\
\hline Original Article & \multirow{10}{*}{$\begin{array}{l}\text { Surveys were conducted in the Nisava and Toplica districts } \\
\text { in Serbia during } 2018 \text {. The medium-sized private sheep } \\
\text { farm (A) in Držanovac in the Toplica District and the small } \\
\text { private sheep farm (B) in Orljan, in the Nisava District } \\
\text { are being investigated. The volume and technology } \\
\text { of sheep genotype production for lamb production for } \\
\text { sale on both farms was monitored and economic results } \\
\text { analyzed. During the study period, it was found that on } \\
\text { farm A there were } 150 \text { heads of Merino breed and on farm } \\
\text { B } 75 \text { heads. Total profit on Farm A without incentives } \\
\text { in crop production: wheat } 1,215.0 \text { EUR, maize } 1329.7 \\
\text { EUR, barley } 1314.60 \text { EUR, triticale } 1561,50 \text { EUR and in } \\
\text { livestock breeding for } 150 \text { heads EUR } 16,920 \text {, of which } \\
13,500 \text { EUR in lamb production. Also total profit on Farm } \\
\text { B in crop production: wheat EUR } 2,853.0 \text {, corn EUR } \\
1329.6 \text { and livestock production, by } 75 \text { heads, EUR } 8,460 \text {, } \\
\text { of which EUR } 6,750 \text { in lamb production. }\end{array}$} \\
\hline Received: 16 December 2019 & \\
\hline Accepted: 05 March 2020 & \\
\hline doi:10.5937/ekoPolj2001125M & \\
\hline UDC 363.3.03:338.43.01 & \\
\hline Keywords: & \\
\hline economic results, production & \\
\hline technology, lambs for sale, body & \\
\hline weight & \\
\hline JEL: $Q 12, Q 13, Q 41$ & \\
\hline
\end{tabular}

(c) 2020 EA. All rights reserved.

\section{Introduction}

The importance of sheep production, among other things, is to enable it to make fuller use of agricultural resources and to realize a large part of crop production. In addition, due to the encompassing production process which has a slight influence of external factors, sheep production significantly affects the overall growth rate of agricultural production. Production of the breed of lambs for the production of lambs for sale has fallen over

1 Ivan D. Mičić, Ph.D., research associate and assistant professor MIT University of Skopje, Bul. Third Macedonian brigades 66a 1000 Skopje, Phone:+381 677059898 , E-mail:divanlav@gmail.com, https://orcid.org/0000-0001-5385-6564

2 Zoran Rajić, Ph.D., full professor, University of Belgrade, Faculty of Agriculture, Nemanjina Street no. 6, 11080 Zemun, Republic of Serbia, Phone:+381 1126153 15, E-mail:zorajic@ agrif.bg.ac.rs, https://orcid.org/0000-0002-1730-2246

3 Ahmet Halilagić, Ph.D. student, University of Belgrade, Faculty of Agriculture, Nemanjina Street no. 6, 11080 Zemun, Republic of Serbia, Phone:+381 1126153 15, E-mail:ahmet. halilagic@hcg.rs, https://orcid.org/0000-0002-7060-4315

http://ea.bg.ac.rs 
one third of the total production in Serbia, while this share in Vojvodina rises to almost half of the total production. Regardless of the natural indicators in intensively oriented production of Merino sheep, it is very important to provide a thorough insight in the cost of production, which is the basis of economy of the production process of lambs for sale. Unlike a farm that produces sheep for its own purposes and eventually sells the product. Farms or family farms that are permanently oriented to commodity production must pay close attention to the cost of production, and the products obtained must meet quality standards. Research on the economic parameters of sheep production is concerned with determining the cost of producing lambs and sheep in two stages. The analysis deals with the cost of production of sheep in the first stage of the production process, and the determination of the total cost of production of individual product categories by applying divisional calculation in the second stage of calculation Mičić et al., (2018). The cost correction aims to give the results obtained wider applicability to the farms surveyed. In this way, the results obtained are of general and not only local importance, Yusup et al., (2017). Quantity, as part of a strategic effort to provide the required quantities of lambs for sale, wool, and milk, continues to be an important element of production, although its primacy has long been overcome, Saatchi et al., (2010). To increase lamb production in the industry, science has made efforts to successfully manage the sheep breeding process. Today, in many countries, including ours, estrus Synchronization methods are used to control the reproductive properties of sheep, as well as to produce more female lambs at the same stage of estrus and ovulation. This method allowed two or three lambs a year for two years, with the goal of increasing lamb and meat production. Zapletal et al., (2010) point out that the use of proper hygiene in sheep reproduction, breeding, environmental conditions, nutrition, prevention and treatment are key factors. Estrus synchronization successful programs play a key role in lambing and profitability of sheep wearers in semi-intensive production systems, (Cividini et al., 2012; Kukovic, et al., 2013). Sheep farming in Serbia is generally extensive. In Serbia the sheep population is $80 \%$, of which the following strains are: Pirot, Svrljiska, Sjenica, while the remaining 20\% are Cigaj, Wirtember sheep, and Australian merino for wool production. Productivity in the population of other breeds is higher, but due to the low participation in the total number of sheep, the effects are insignificant at the national level. The annual weight of sheepmeat in the last decade is below 20,000 tonnes. In Serbia, the consumption of sheep meat is below $3.0 \mathrm{~kg}$ per capita, we are among the European countries with the lowest consumption. The basic orientation of farmers in lamb and meat production in Serbia seems to be in the first (F1) generation to achieve better quality of lamb production for sale, etc. According to the latest data from the Statistical Office of the Republic, about 1.7 million sheep are raised. Most, over one million, are grown in central and eastern Serbia, but on the other hand, the largest and most organized farms are located in Vojvodina (Matsushita et al., 2010; Marina et al., 2017).

The following authors have explored this issue, among others: (Kegalj et al., 2011; Mellado et al., 2016; Momoh et al., 2013; Rahimi et al., 2014; Siddalingamurthy et al., 2017; Simeonov et al. al., 2015; Tohidi et al., 2016; Catalan et al., 2018). 


\section{Materials and methods}

The survey was conducted on a family farm on farm A in Držanovac and farm B in Orljan. Both farms have a closed production cycle. The middle farm (A) has 150 heads of sheep, and the small farm (B) 75 heads of sheep a year. Farm A employed 2 people and Farm B 1 people. Production costs for both households are based on natural indicators established on the basis of a survey conducted in 2018 and all categories of variable costs in accordance with the production process. Material costs refer to the consumption of nutrients and medicines used in the production process. Depreciation expense in 2018 based on space and equipment norms, we approach investment estimates, estimate depreciation costs based on which fixed cost categories are calculated. In determining the cost of production, we start from the price of lamb products for sale. Production parameters are monitored: on farms $\mathrm{A}$ and $\mathrm{B}$, food consumption on both farms per $1 \mathrm{~kg}$ increment, total growth and food costs on both farms Gbangboche et al., (2006).

The significance of results in lamb production in 2018 was independently monitored on both farms during one research year.

\section{Results}

Research on the economic parameters of the production process for lambs for sale was carried out at Medium Sheep Farm A and Small Sheep Farm B. These farms have a closed production cycle which includes the production of lambs, sheep wool and milk. On farm A over 200 lambs a year and farm B about 100 lambs. Fama A produces the most important nutrients for the feeding of cereals. at $10 \mathrm{~h}$ and Fama B produces the most important nutrients for the feeding of the cereals, at $5 \mathrm{~h}$. Farm A has two members and Farm $\mathrm{B}$ has one member. Based on the recording of production processes on farms $\mathrm{A}$ and $\mathrm{B}$, the cost of materials was calculated, which included the consumption of food, medicines, other materials and water. Variable costs account for the bulk of material costs (Archimede et al., 2008; Zaharia et al., 2013).

\section{Discussions}

Farm A was monitored for the economics of producing the most important nutrients for the feeding of the cereal group. The economics of wheat, maize, triticale and barley production are still being monitored. The farmer has significant areas and favorable conditions for the said production. Corn covered 2 ha, triticale 3 ha, wheat 2 ha and barley 3 ha. Annual production of 50 tonnes of cereals on farm A farmland in 2018 . The aforementioned middle sheep farm as well as crop production was investigated in order to provide nutrients for the feeding of herds on farm A. The natural and financial indicators of cereal production in 2018 are given in the following tables, as follows: calculation of production, maize (Table 1 ); triticale (Table 2); wheat (Table 3) and barley (Table 4). 
Table 1: Calculation of realized economic indicators of maize production per 2 ha for farm A

\begin{tabular}{|c|c|c|c|c|c|c|c|}
\hline $\begin{array}{l}\text { Row. } \\
\text { Num } \\
\text { ber } \\
\end{array}$ & Production year: 2018 & \multirow{2}{*}{$\begin{array}{l}\text { Number } \\
\text { of } \\
\text { repetitions }\end{array}$} & \multirow[t]{2}{*}{ Quantity } & \multirow[t]{2}{*}{ JM } & \multirow[t]{2}{*}{$\begin{array}{l}\text { The } \\
\text { price }\end{array}$} & \multirow[t]{2}{*}{$\mathbf{J M}$} & \multirow[t]{2}{*}{$\begin{array}{l}\text { Amount of } \\
\text { EUR / ha / } 2\end{array}$} \\
\hline $\mathbf{I}$ & Income & & & & & & \\
\hline 1. & Corn & 2 & 6,5 & $\mathrm{t} / \mathrm{ha}$ & 130,0 & $\mathrm{EUR} / \mathrm{t}$ & 1690,0 EUR \\
\hline 2. & Corn & 2 & 9 & $\mathrm{t} / \mathrm{ha}$ & 17,98 & $\mathrm{EUR} / \mathrm{t}$ & 323,7 EUR \\
\hline A) & Total revenue (1 to 2 ) & & & & & & 2.013,7 EUR \\
\hline 3. & Costs & & & & & & \\
\hline 4. & Seeds & 1 & 20 & $\mathrm{~kg}$ & 1,50 & EUR & 30,0 EUR \\
\hline 5. & Fertilizer & & & & & & \\
\hline 6. & Manure & $25 \%$ & 40 & $\mathrm{t}$ & 1,0 & $\mathrm{EUR} / \mathrm{kg}$ & 40,0 EUR \\
\hline 7. & KAN $(29 \% \mathrm{~N})$ & & 400 & $\mathrm{~kg}$ & 0,30 & $\mathrm{EUR} / \mathrm{kg}$ & 120,0 EUR \\
\hline 8. & Pesticides & & & & & & \\
\hline 9. & Guardian & & 6 & $\mathrm{~L}$ & 4,0 & $\mathrm{EUR} / \mathrm{kg}$ & 18,0 EUR \\
\hline 10. & Thesis & & 6 & $\mathrm{~L}$ & 2,5 & EUR /L & 15,0 EUR \\
\hline 11. & Irrigation & & & & & & \\
\hline 12. & Energent & 2 & 15 & $\mathrm{~L}$ & 1,40 & EUR /L & 42,0 EUR \\
\hline 13. & Diesel fuel & & 60 & $\mathrm{~L}$ & 1,40 & EUR /L & 84,0 EUR \\
\hline 14. & $\begin{array}{l}\text { Maintenance of } \\
\text { mechanization }\end{array}$ & & 2 & ha & 15,0 & EUR /ha & 30,0 EUR \\
\hline 15. & $\begin{array}{l}\text { Paid services. } \\
\text { mechanization }\end{array}$ & & & & & & \\
\hline 16. & Plowing & & 2 & ha & & EUR /ha & 0 EUR \\
\hline 17. & Land preparation & & 2 & ha & & EUR/ha & 0 EUR \\
\hline 18. & Sowing & & 2 & ha & 30.50 & EUR /ha & 61,0 EUR \\
\hline 19. & Harvest & & 2 & ha & 65,00 & EUR /ha & 130,0 EUR \\
\hline 20. & Paid labor & & 5 & $\begin{array}{l}\text { work } \\
\text { day }\end{array}$ & 15,0 & EUR & 75,0 EUR \\
\hline 21. & Other variable costs & & & & & & \\
\hline 22. & Storage cost & & & $\mathrm{kg}$ & & $\mathrm{EUR} / \mathrm{kg}$ & 0 EUR \\
\hline 23. & Transport to the customer & & 13 & $\mathrm{t}$ & 3,0 & $\mathrm{EUR} / \mathrm{t}$ & 39,0 EUR \\
\hline В) & \multicolumn{2}{|l|}{ Total Cost (3 to 23) } & & & & & 684,0 EUR \\
\hline II & \multicolumn{2}{|l|}{ PROFIT / LOSS } & & & & & \\
\hline 24. & \multicolumn{2}{|l|}{ Total No Incentives (A - B) } & & & & & 1329,7 EUR \\
\hline 25. & \multicolumn{2}{|c|}{ Per ha without incentives $(24: 17)$} & & & & & 664,8 EUR \\
\hline 26. & \multicolumn{2}{|l|}{ Price of cereals $\mathrm{kg}(24: 1)$} & & & & & 0,1 EUR \\
\hline 27. & \multicolumn{2}{|c|}{ Economical production (A: B) } & & & & & 2,94 \\
\hline 28. & \multicolumn{2}{|c|}{ Production profitability (24: B) x100 } & & & & & $194,40 \%$ \\
\hline
\end{tabular}

Source: Mičić, 2018 
Results achieved: The average maize yield on farm A was $6.5 \mathrm{t} / \mathrm{ha}$, ranging from 6.0 $\mathrm{t} /$ ha to $7.0 \mathrm{t} / \mathrm{ha}$. Total profit per 2 ha is EUR 1,329.70, production efficiency is 2.94 and production profitability is $19.40 \%$.

Table 2: Calculation of realized economic indicators of triticale production per 3 ha for farm A

\begin{tabular}{|c|c|c|c|c|c|c|c|}
\hline $\begin{array}{l}\text { Row. } \\
\text { Num } \\
\text { ber }\end{array}$ & Production year: 2018 & \multirow{2}{*}{$\begin{array}{l}\text { Num- } \\
\text { ber of } \\
\text { repeti- } \\
\text { tions }\end{array}$} & \multirow[t]{2}{*}{$\begin{array}{l}\text { Qua } \\
\text { ntity }\end{array}$} & \multirow[t]{2}{*}{$\mathbf{J M}$} & \multirow[t]{2}{*}{$\begin{array}{l}\text { The } \\
\text { price }\end{array}$} & \multirow[t]{2}{*}{$\mathbf{J M}$} & \multirow[t]{2}{*}{$\begin{array}{l}\text { Amount of } \\
\text { EUR/ha/3 }\end{array}$} \\
\hline $\mathbf{I}$ & Income & & & & & & \\
\hline 1. & Triticale & 3 & 5,0 & $\mathrm{t} / \mathrm{ha}$ & 150,0 & $\mathrm{EUR} / \mathrm{t}$ & 2.250,0 EUR \\
\hline 2. & Straw & 3 & 5 & $\mathrm{t} / \mathrm{ha}$ & 19,5 & EUR /t & 292,5 EUR \\
\hline A) & Total revenue (1 to 2 ) & & & & & & 2.542,5EUR \\
\hline 3 & Costs & & & & & & \\
\hline 4. & Seeds & 1 & 750 & $\mathrm{~kg}$ & 0,20 & EUR & 150,00 EUR \\
\hline 5. & Fertilizer & & & & & & \\
\hline 6. & Manure & $25 \%$ & 60,0 & $\mathrm{~kg}$ & 1,0 & EUR/kg & 60,00 EUR \\
\hline 7. & Urea & & 600 & $\mathrm{~kg}$ & 0,30 & EUR $/ \mathrm{kg}$ & 180,00 EUR \\
\hline 8. & Foliar savings & & 6 & $\mathrm{~kg}$ & 3,0 & EUR/kg & 18,00 EUR \\
\hline 9. & Pesticides & & & & & & \\
\hline 10. & Meteor & & 30 & G & 0,15 & EUR /L & 4,50 EUR \\
\hline 11. & Irrigation & & & & & & \\
\hline 12. & Energent & 3 & 15 & $\mathrm{~L}$ & 1,40 & EUR /L & 63,00 EUR \\
\hline 13. & Diesel fuel & & 90 & $\mathrm{~L}$ & 1,40 & EUR/L & 126,00 EUR. \\
\hline 14. & Maintenance of mechanization & & 3 & ha & 19,00 & EUR /ha & 57,00 EUR \\
\hline 15. & Paid services. mechanization & & & & & & \\
\hline 16. & Plowing & & 3 & ha & & EUR /ha & 0 EUR \\
\hline 17. & Land preparation & & 3 & ha & & EUR/ha & 0 EUR \\
\hline 18. & Sowing & & 3 & ha & 30,5 & EUR /ha & 91,50 EUR \\
\hline 19. & Harvest & & 3 & ha & 52,00 & EUR/ha & 156,00 EUR \\
\hline 20. & Paid seasonal labor & & 20 & $\begin{array}{l}\text { work } \\
\text { day }\end{array}$ & 1,50 & EUR & 30,00 EUR \\
\hline 21. & Other variable costs & & & & & & \\
\hline 22. & Storage cost & & & $\mathrm{kg}$ & & EUR/kg & 0 EUR \\
\hline 23. & Transport to the customer & & 15,0 & $\mathrm{t}$ & 3,0 & $\mathrm{EUR} / \mathrm{kg}$ & 45 EUR \\
\hline B) & Total Cost (3 to 23) & & & & & & 981,0 EUR \\
\hline II & PROFIT / LOSS & & & & & & \\
\hline 24. & Total No Incentives (A - B) & & & & & & 1561,50 EUR \\
\hline 25. & Per ha without incentives $(24: 17)$ & & & & & & $\mathbf{5 2 0 , 5 0}$ EUR \\
\hline 26. & Price of cereals kg $(24: 1)$ & & & & & & $\mathbf{0 , 1 0}$ EUR \\
\hline 27. & Economical production (A: B) & & & & & & 2,59 \\
\hline 28. & Production profitability $(24: \mathrm{B}) \mathrm{x}$ & 100 & & & & & $159,17 \%$ \\
\hline
\end{tabular}

Source: Mičić, 2018 
Achieved results: The average yield of triticale on the tested farm A was $5.0 \mathrm{t} / \mathrm{ha}$, and ranged from $4.5 \mathrm{t} /$ ha to $5.5 \mathrm{t} /$ ha. Total profit per 3 ha is EUR 1,561.50, production efficiency is 2.59 and production profitability is $159.17 \%$.

Table 3: Calculation of realized economic indicators of wheat production per 2 ha for farm A

\begin{tabular}{|c|c|c|c|c|c|c|c|}
\hline $\begin{array}{l}\text { Row. } \\
\text { Num } \\
\text { ber }\end{array}$ & $\begin{array}{l}\text { Production year: } \\
2018\end{array}$ & \multirow{2}{*}{$\begin{array}{l}\text { Num- } \\
\text { ber of } \\
\text { repeti } \\
\text { tions }\end{array}$} & \multirow[t]{2}{*}{$\begin{array}{l}\text { Quan- } \\
\text { tity }\end{array}$} & \multirow[t]{2}{*}{$\mathbf{J M}$} & \multirow[t]{2}{*}{$\begin{array}{l}\text { The } \\
\text { price }\end{array}$} & \multirow[t]{2}{*}{$\mathbf{J M}$} & \multirow[t]{2}{*}{$\begin{array}{l}\text { Amount of } \\
\text { EUR/ha/2 }\end{array}$} \\
\hline I & Income & & & & & & \\
\hline 1. & Wheat & 2 & 5,0 & t/ha & 170,0 & $\begin{array}{l}\text { EUR } \\
/ \mathrm{t}\end{array}$ & $\begin{array}{r}1700,0 \\
\text { EUR }\end{array}$ \\
\hline 2. & Straw & 2 & 5.0 & t/ha & 16,9 & $\mathrm{EUR} / \mathrm{t}$ & 169,0 EUR \\
\hline A) & Total revenue ( 1 to 2 ) & & & & & & $\begin{array}{l}1.869,0 \\
\text { EUR } \\
\end{array}$ \\
\hline 3 & Costs & & & & & & \\
\hline 4. & Seeds & 1 & 500 & $\mathrm{~kg}$ & 0,20 & EUR & $\begin{array}{r}100,00 \\
\text { EUR }\end{array}$ \\
\hline 5. & Fertilizer & & & & & & \\
\hline 6. & Manure & $25 \%$ & 40 & $\mathrm{t}$ & 1,0 & $\begin{array}{l}\text { EUR / } \\
\mathrm{kg}\end{array}$ & 40,00 EUR \\
\hline 7. & Urea & & 400 & $\mathrm{~kg}$ & 0,30 & $\begin{array}{l}\mathrm{EUR} / \\
\mathrm{kg}\end{array}$ & $\begin{array}{r}120,00 \\
\text { EUR }\end{array}$ \\
\hline 8. & Foliar savings & & 4 & $\mathrm{~kg}$ & 3,0 & $\begin{array}{l}\mathrm{EUR} / \\
\mathrm{kg}\end{array}$ & 12,00 EUR \\
\hline 9. & Pesticides & & & & & & \\
\hline 10. & Meteor & & 20 & $\mathrm{~g}$ & 0,15 & EUR /L & 3,0 EUR \\
\hline 11. & Irrigation & & & & & & \\
\hline 12. & Energent & 2 & 15 & $\mathrm{~L}$ & 1,40 & EUR/L & 42,00 EUR \\
\hline 13. & Diesel fuel & & 60 & $\mathrm{~L}$ & 1,40 & $\mathrm{EUR} / \mathrm{L}$ & 84,00 EUR \\
\hline 14. & $\begin{array}{l}\text { Maintenance of mech- } \\
\text { anization }\end{array}$ & & 2 & ha & 19,0 & $\begin{array}{l}\text { EUR / } \\
\text { ha }\end{array}$ & $38,00 \mathrm{EUR}$ \\
\hline 15. & $\begin{array}{l}\text { Paid services. mecha- } \\
\text { nization }\end{array}$ & & & & & & \\
\hline 16. & Plowing & & 2 & ha & & EUR/g & 0 EUR \\
\hline 17. & Land preparation & & 2 & ha & & $\begin{array}{l}\text { EUR / } \\
\text { ha }\end{array}$ & 0 EUR \\
\hline 18. & Sowing & & 2 & ha & 30,0 & $\begin{array}{l}\text { EUR / } \\
\text { ha }\end{array}$ & 60,0 EUR \\
\hline 19. & Harvest & & 2 & ha & 55,0 & $\begin{array}{l}\text { EUR / } \\
\text { ha }\end{array}$ & $\begin{array}{r}110,00 \\
\text { EUR }\end{array}$ \\
\hline
\end{tabular}




\begin{tabular}{|c|c|c|c|c|c|c|c|}
\hline $\begin{array}{l}\text { Row. } \\
\text { Num } \\
\text { ber }\end{array}$ & $\begin{array}{l}\text { Production year: } \\
2018\end{array}$ & \multirow{2}{*}{$\begin{array}{l}\text { Num- } \\
\text { ber of } \\
\text { repeti } \\
\text { tions }\end{array}$} & \multirow[t]{2}{*}{$\begin{array}{l}\text { Quan- } \\
\text { tity }\end{array}$} & \multirow[t]{2}{*}{$\mathbf{J M}$} & \multirow[t]{2}{*}{$\begin{array}{l}\text { The } \\
\text { price }\end{array}$} & \multirow[t]{2}{*}{$\mathbf{J M}$} & \multirow[t]{2}{*}{$\begin{array}{l}\text { Amount of } \\
\text { EUR/ha/2 }\end{array}$} \\
\hline $\mathbf{I}$ & Income & & & & & & \\
\hline 20. & $\begin{array}{l}\text { Paid season. work- } \\
\text { force }\end{array}$ & & 10 & $\begin{array}{l}\text { work } \\
\text { day }\end{array}$ & 1,50 & EUR & $15,0 \mathrm{EUR}$ \\
\hline 21. & Other variable costs & & & & & & \\
\hline 22. & Storage cost & & & $\mathrm{kg}$ & & $\begin{array}{l}\text { EUR / } \\
\mathrm{kg}\end{array}$ & 0 EUR \\
\hline 23. & $\begin{array}{l}\text { Transport to the cus- } \\
\text { tomer }\end{array}$ & & 10 & $\mathrm{t}$ & 0,3 & $\begin{array}{l}\text { EUR / } \\
\mathrm{kg}\end{array}$ & 30,0 EUR \\
\hline B) & \multicolumn{2}{|l|}{ Total Cost (3 to 23) } & & & & & 654,0 EUR \\
\hline II & \multicolumn{2}{|l|}{ PROFIT / LOSS } & & & & & \\
\hline 24. & \multicolumn{2}{|c|}{ Total No Incentives (A - B) } & & & & & $\begin{array}{l}\mathbf{1 . 2 1 5 , 0} \\
\text { EUR }\end{array}$ \\
\hline 25. & \multicolumn{2}{|c|}{ Per ha out of reach $(24: 17)$} & & & & & $\begin{array}{r}\mathbf{6 0 7 , 5 0} \\
\text { EUR }\end{array}$ \\
\hline 26. & \multicolumn{2}{|c|}{ Price of cereals kg $(24: 1)$} & & & & & $\mathbf{0 , 1 2}$ EUR \\
\hline 27. & \multicolumn{2}{|c|}{ Economical production (A: B) } & & & & & 2,86 \\
\hline 28. & \multicolumn{2}{|c|}{$\begin{array}{l}\text { Production profitability (24: B) } \\
\text { x } 100\end{array}$} & & & & & $185,78 \%$ \\
\hline
\end{tabular}

Source: Mičić, 2018

Results achieved: The average wheat yield on farm A tested was $5.0 \mathrm{t} / \mathrm{ha}$, ranging from $4.5 \mathrm{t} /$ ha to $5.5 \mathrm{t} /$ ha. Total profit per 2 ha is EUR 1,215.0, production efficiency is 2.86 and production profitability is $185.78 \%$.

Table 4: Calculation of economic indicators of barley production per 3 ha for farm A

\begin{tabular}{|c|l|r|r|r|r|r|r|}
\hline $\begin{array}{l}\text { Row. } \\
\text { Num } \\
\text { ber }\end{array}$ & Production year: 2018 & $\begin{array}{l}\text { Num- } \\
\text { ber of } \\
\text { repeti } \\
\text { tions }\end{array}$ & $\begin{array}{l}\text { Qua } \\
\text { ntity }\end{array}$ & JM & $\begin{array}{l}\text { The } \\
\text { price }\end{array}$ & JM & $\begin{array}{c}\text { Amount of } \\
\text { EUR/ha/3 }\end{array}$ \\
\hline I & Income & 3 & 4,0 & $\mathrm{t} / \mathrm{ha}$ & 170,0 & EUR $/ \mathrm{t}$ & $2.040,0 \mathrm{EUR}$ \\
\hline 1. & Barley & 3 & 5 & $\mathrm{t} / \mathrm{ha}$ & 17,04 & $\mathrm{EUR} / \mathrm{t}$ & $255,6 \mathrm{EUR}$ \\
\hline 2. & Straw & & & & & & $2.295,6 \mathrm{EUR}$ \\
\hline A) & Total revenue (1 to 2) & & & & & & \\
\hline $\mathbf{3}$ & Costs & 1 & 750 & $\mathrm{~kg}$ & 0,20 & $\mathrm{EUR}$ & $150,00 \mathrm{EUR}$ \\
\hline 4. & Seeds & & & & & & \\
\hline 5. & Fertilizer & $25 \%$ & 60,0 & $\mathrm{t}$ & 1,0 & $\mathrm{EUR} / \mathrm{kg}$ & $60,00 \mathrm{EUR}$ \\
\hline 6. & Manure & & 600 & $\mathrm{~kg}$ & 0,3 & $\mathrm{EUR} / \mathrm{kg}$ & $180,00 \mathrm{EUR}$ \\
\hline 7. & Urea & & 6 & $\mathrm{~kg}$ & 3,0 & $\mathrm{EUR} / \mathrm{kg}$ & $18,0 \mathrm{EUR}$ \\
\hline 8. & Foliar savings & & & & & & \\
\hline 9. & Pesticides & & & \\
\hline
\end{tabular}




\begin{tabular}{|c|c|c|c|c|c|c|c|}
\hline $\begin{array}{l}\text { Row. } \\
\text { Num } \\
\text { ber }\end{array}$ & Production year: 2018 & \multirow{2}{*}{$\begin{array}{l}\text { Num- } \\
\text { ber of } \\
\text { repeti } \\
\text { tions } \\
\end{array}$} & \multirow[t]{2}{*}{$\begin{array}{l}\text { Qua } \\
\text { ntity }\end{array}$} & \multirow[t]{2}{*}{$\mathbf{J M}$} & \multirow[t]{2}{*}{$\begin{array}{l}\text { The } \\
\text { price }\end{array}$} & \multirow[t]{2}{*}{$\mathbf{J M}$} & \multirow[t]{2}{*}{$\begin{array}{l}\text { Amount of } \\
\text { EUR/ha/3 }\end{array}$} \\
\hline $\mathbf{I}$ & Income & & & & & & \\
\hline 10. & Meteor & & 30 & $\mathrm{~g}$ & 0,15 & EUR/L & 4,50 EUR \\
\hline 11. & Irrigation & & & & & & \\
\hline 12. & Energent & 3 & 15 & $\mathrm{~L}$ & 1,40 & EUR/L & 63,0 EUR \\
\hline 13. & Diesel fuel & & 90 & $\mathrm{~L}$ & 1,40 & $\mathrm{EUR} / \mathrm{L}$ & 126,00 EUR \\
\hline 14. & Maintenance of mechanization & & 3 & ha & 19,0 & EUR /ha & 57,0 EUR \\
\hline 15. & Paid services. mechanization & & & & & & \\
\hline 16. & Plowing & & & ha & & EUR /ha & 0 EUR \\
\hline 17. & Land preparation & & 3 & ha & & EUR /ha & 0 EUR \\
\hline 18. & Sowing & & 3 & ha & 30,50 & EUR /ha & 91,50 EUR \\
\hline 19. & Harvest & & 3 & ha & 55,00 & EUR /ha & 165,0 EUR \\
\hline 20. & Paid season. workforce & & 20 & \begin{tabular}{|l} 
work \\
day
\end{tabular} & 1,50 & EUR & 30,0 EUR \\
\hline 21. & Other variable costs & & & & & & \\
\hline 22. & Storage cost & & & $\mathrm{kg}$ & & $\mathrm{EUR} / \mathrm{kg}$ & 0 EUR \\
\hline 23. & Transport to the customer & & 12 & $\mathrm{t}$ & 3,0 & $\mathrm{EUR} / \mathrm{kg}$ & 36,0 EUR \\
\hline B) & \multicolumn{2}{|l|}{ Total Cost (3 to 23) } & & & & & 981,0 EUR \\
\hline II & \multicolumn{2}{|l|}{ PROFIT / LOSS } & & & & & \\
\hline 24. & \multicolumn{2}{|l|}{ Total No Incentives (A - B) } & & & & & 1314,60 EUR \\
\hline 25. & \multicolumn{2}{|l|}{ Per ha without incentives $(24: 17)$} & & & & & 438,20 EUR \\
\hline $\begin{array}{l}\text { Row. } \\
\text { Num } \\
\text { ber }\end{array}$ & \multicolumn{2}{|l|}{ Production year: 2018} & $\begin{array}{l}\text { Num- } \\
\text { ber of } \\
\text { repeti } \\
\text { tions } \\
\end{array}$ & $\begin{array}{l}\text { Qua } \\
\text { ntity }\end{array}$ & $\mathbf{J M}$ & The price & $\mathbf{J M}$ \\
\hline 26. & \multicolumn{2}{|l|}{ Price of cereals kg (24: 1$)$} & & & & & 0,11 EUR \\
\hline 27. & \multicolumn{2}{|l|}{ Economical production (A: B) } & & & & & 2,34 \\
\hline 28. & \multicolumn{2}{|c|}{ Production profitability (24: B) x 100} & & & & & $134,00 \%$ \\
\hline
\end{tabular}

Source: Mičić, 2018

Results achieved: The average yield of barley on farm A was $4.0 \mathrm{t} / \mathrm{ha}$, ranging from $3.5 \mathrm{t} /$ ha to $4.5 \mathrm{t} / \mathrm{ha}$. Total profit on 3 ha is EUR 1,314.60, production efficiency is 2.34 and profitability of production is $134.0 \%$

The economics of feed production for the herd feed were also monitored on the sheep farm B from the cereals group: maize and wheat. The farm has the conditions and areas for crop production. Corn covers 2 ha and wheat 3 ha. Farm B's annual cereal production is $35.5 \mathrm{t}$ in 2018 .

The natural and financial indicators are presented in the table below: the calculation of realized economic production, maize (Table 5) and wheat (Table 6). 
Economics of Agriculture, Year 67, No. 1, 2020, (pp. 125-139), Belgrade

Table 5: Calculation of realized economic indicators of maize production per 2 ha for farm B

\begin{tabular}{|c|c|c|c|c|c|c|c|}
\hline $\begin{array}{l}\text { Row. } \\
\text { Num } \\
\text { ber }\end{array}$ & Production year: 2018 & \multirow{2}{*}{\begin{tabular}{|l|} 
Num- \\
ber of \\
repeti \\
tions \\
\end{tabular}} & \multirow[t]{2}{*}{$\begin{array}{l}\text { Qua } \\
\text { ntity }\end{array}$} & \multirow[t]{2}{*}{$\mathbf{J M}$} & \multirow[t]{2}{*}{$\begin{array}{c}\text { The } \\
\text { price }\end{array}$} & \multirow[t]{2}{*}{$\mathbf{J M}$} & \multirow[t]{2}{*}{$\begin{array}{l}\text { Amount of } \\
\text { EUR/ha/2 }\end{array}$} \\
\hline I & Income & & & & & & \\
\hline 1. & Corn & 2 & 6,5 & $\mathrm{t} / \mathrm{ha}$ & 130,0 & $\mathrm{EUR} / \mathrm{t}$ & 1690,0 EUR \\
\hline 2. & Corn & 2 & 9 & $\mathrm{t} / \mathrm{ha}$ & 17,98 & $\mathrm{EUR} / \mathrm{t}$ & 323,6 EUR \\
\hline A) & Total revenue (1 to 2 ) & & & & & & $2.013,6$ EUR \\
\hline 3. & Costs & & & & & & \\
\hline 4. & Seeds & 1 & 20 & $\mathrm{~kg}$ & 1,50 & EUR & 30,0 EUR \\
\hline 5. & Fertilizer & & & & & & \\
\hline 6. & Manure & $25 \%$ & 40 & $\mathrm{t}$ & 1,0 & $\mathrm{EUR} / \mathrm{kg}$ & 40,0 EUR \\
\hline 7. & $\operatorname{KAN}(29 \% \mathrm{~N})$ & & 400 & $\mathrm{~kg}$ & 0,30 & $\mathrm{EUR} / \mathrm{kg}$ & 120,0 EUR \\
\hline 8. & Pesticides & & & & & & \\
\hline 9. & Guardian & & 6 & $\mathrm{~L}$ & 4,0 & $\mathrm{EUR} / \mathrm{kg}$ & 18,0 EUR \\
\hline 10. & Thesis & & 6 & $\mathrm{~L}$ & 2,5 & EUR /L & 15,0 EUR \\
\hline 11. & Irrigation & & & & & & \\
\hline 12. & Energent & 2 & 15 & $\mathrm{~L}$ & 1,40 & $\mathrm{EUR} / \mathrm{L}$ & 42,0 EUR \\
\hline 13. & Diesel fuel & & 60 & $\mathrm{~L}$ & 1,40 & EUR /L & 84,0 EUR \\
\hline 14. & $\begin{array}{l}\text { Maintenance of mechaniza- } \\
\text { tion }\end{array}$ & & 2 & ha & 15,0 & EUR /ha & 30,0 EUR \\
\hline 15. & Paid usl. mechanization & & & & & & \\
\hline $\begin{array}{l}\text { Row. } \\
\text { Num } \\
\text { ber }\end{array}$ & Production year: 2018 & $\begin{array}{l}\text { Num- } \\
\text { ber of } \\
\text { repeti } \\
\text { tions }\end{array}$ & $\begin{array}{l}\text { Qua } \\
\text { ntity }\end{array}$ & $\mathbf{J M}$ & $\begin{array}{l}\text { The } \\
\text { price }\end{array}$ & $\mathbf{J M}$ & $\begin{array}{l}\text { Amount of } \\
\text { EUR/ha/3 }\end{array}$ \\
\hline 16. & Plowing & & 2 & ha & & EUR /ha & 0 EUR \\
\hline 17. & Land preparation & & 2 & ha & & EUR /ha & 0 EUR \\
\hline 18. & Sowing & & 2 & ha & 30.50 & EUR/ha & 61,0 EUR \\
\hline 19. & Harvest & & 2 & ha & 65,0 & EUR /ha & 130,0 EUR \\
\hline 20. & Paid labor & & 5 & $\begin{array}{l}\text { work } \\
\text { day }\end{array}$ & 15,0 & EUR & 75,0 EUR \\
\hline 21. & Other variable costs & & & $\mathrm{kg}$ & & $\mathrm{EUR} / \mathrm{kg}$ & \\
\hline 22. & Storage cost & & & & & & \\
\hline 23. & Transport to the customer & & 13 & $\mathrm{t}$ & 3,0 & $\mathrm{EUR} / \mathrm{t}$ & 39,0 EUR \\
\hline B) & \multicolumn{2}{|l|}{ Total Cost (3 to 23) } & & & & & 684,0 EUR \\
\hline II & \multicolumn{2}{|l|}{ PROFIT / LOSS } & & & & & \\
\hline 24. & \multicolumn{2}{|l|}{ Total No Incentives (A - B) } & & & & & 1329,6 EUR \\
\hline 25. & \multicolumn{2}{|c|}{ Per ha without incentives $(24: 17)$} & & & & & 664,8 EUR \\
\hline 26. & \multicolumn{2}{|l|}{ Price of cereals kg (24: 1$)$} & & & & & 0,1 EUR \\
\hline 27. & \multicolumn{2}{|l|}{ Economical production (A: B) } & & & & & 2,94 \\
\hline 28. & \multicolumn{2}{|c|}{ Production profitability (24: B) x100 } & & & & & $194,40 \%$ \\
\hline
\end{tabular}

Source: Mičić, 2018 
Results achieved: The average maize yield on farm $\mathrm{B}$ tested was $6.5 \mathrm{t} / \mathrm{ha}$, ranging from $6.0 \mathrm{t} /$ ha to $7.0 \mathrm{t} / \mathrm{ha}$.

Total profit per 2 ha is EUR 1,329.70, production efficiency is 2.94 and production profitability is $19.40 \%$.

Table 6: Calculation of realized economic indicators of wheat production per 3 ha for farm B

\begin{tabular}{|c|c|c|c|c|c|c|c|}
\hline $\begin{array}{l}\text { Row. } \\
\text { Num } \\
\text { ber } \\
\end{array}$ & Production year 2018 & $\begin{array}{l}\text { Num- } \\
\text { ber of } \\
\text { repeti }\end{array}$ & $\begin{array}{l}\text { Qua } \\
\text { ntity }\end{array}$ & $\mathbf{J M}$ & $\begin{array}{l}\text { The } \\
\text { price }\end{array}$ & $\mathbf{J M}$ & $\begin{array}{l}\text { Amount of } \\
\text { EUR/ha/2 }\end{array}$ \\
\hline I & Income & tions & & & & & \\
\hline 1. & Simonida wheat of 3 ha & 3 & 7,5 & $\mathrm{t} / \mathrm{ha}$ & 170,0 & $€ / \mathrm{t}$ & $3.825,0$ \\
\hline 2. & 3 ha straw & 3 & 4 & $\mathrm{t} / \mathrm{ha}$ & 8,75 & $€ / \mathrm{t}$ & 105,0 \\
\hline 3 & $\begin{array}{l}\text { RS incentives for crop produc- } \\
\text { tion }\end{array}$ & & 3 & ha & 50,00 & $€ /$ ha & 150,0 \\
\hline A) & Total income (1 to 2 ) for 3 ha & & & & & & $4.080,0$ \\
\hline 4 & Costs & & & & & & \\
\hline 5. & Seed for 3 ha & 1 & 750 & $\mathrm{~kg}$ & 0,20 & $\mathrm{~kg}$ & 150,0 \\
\hline 6. & Fertilizer & & & & & & \\
\hline 7. & Manure for $3 \mathrm{ha}$ & & 15 & $\mathrm{t}$ & 4,00 & $\mathrm{t}$ & 60,0 \\
\hline 8. & KAN $(29 \%$ N) $50 \%+$ Urea $50 \%$ & & 1500 & $\mathrm{~kg}$ & 0,30 & $\mathrm{~kg}$ & 450,0 \\
\hline 9. & Foliar savings & & 6 & $\mathrm{~kg}$ & 3,00 & $\mathrm{~kg}$ & 18,0 \\
\hline $\begin{array}{l}\text { Row. } \\
\text { Num } \\
\text { ber }\end{array}$ & Production year: 2018 & $\begin{array}{l}\text { Num- } \\
\text { ber of } \\
\text { repeti } \\
\text { tions } \\
\end{array}$ & $\begin{array}{l}\text { Qua } \\
\text { ntity }\end{array}$ & $\mathbf{J M}$ & $\begin{array}{l}\text { The } \\
\text { price }\end{array}$ & $\mathbf{J M}$ & $\begin{array}{l}\text { Amount of } \\
\text { EUR/ha/3 }\end{array}$ \\
\hline 10 & Pesticides & & & & & & \\
\hline 11 & Meteor & & 30 & $\mathrm{~g}$ & 0,20 & $€ / \mathrm{L}$ & 6,0 \\
\hline 12 & Irrigation & & & & & & \\
\hline 13 & Energy for 3 ha & 3 & 15 & $\mathrm{~L}$ & 1,20 & $€ / \mathrm{L}$ & 54,0 \\
\hline 14 & Diesel fuel & & 90 & $\mathrm{~L}$ & 1,20 & $€ / \mathrm{L}$ & 108,0 \\
\hline 15 & Maintenance of mechanization & & 3 & ha & 19,00 & $€ /$ ha & 57,0 \\
\hline 16 & Paid services & & & ha & & & \\
\hline 17 & Plowing & & 3 & ha & & $€ /$ ha & 0 \\
\hline 18 & Land preparation & & 3 & ha & & $€ /$ ha & 0 \\
\hline 19 & Sowing & & $3 /$ & & 30,00 & $€ /$ ha & 90,0 \\
\hline 20 & Harvest & & 3 & ha & 55,00 & $€ /$ ha & 165,0 \\
\hline 21 & Paid seasonal labor & & 46 & \begin{tabular}{|l|} 
work \\
day
\end{tabular} & 1,50 & $€ / \mathrm{h}$ & 69,0 \\
\hline 22 & Other variable costs & & & & & & \\
\hline 23 & Storage cost & & & $€ / \mathrm{t}$ & & $€ / \mathrm{t}$ & 0 \\
\hline 24 & Transport to the customer & & & $\mathrm{t}$ & & $€ / \mathrm{t}$ & 0 \\
\hline B) & Total Cost (3 to 24) & & & & & & $1.227,0$ \\
\hline II & PROFIT / LOSS & & & & & & \\
\hline 25 & Total with Incentive (A - B) & & & & & & $2.853,0$ \\
\hline 26 & Per ha from incentives $(25: 17)$ & & & & & & 951,0 \\
\hline
\end{tabular}




\begin{tabular}{|l|l|l|l|l|r|r|}
\hline 27 & Cost per kg of grain grain (B: 1) & & & & & $\mathbf{0 , 0 5 4 5}$ \\
\hline 28 & Economical production (A: B) & & & & & $\mathbf{3 , 3 3}$ \\
\hline 29 & Income Profitability (25: A) x 100 & & & & & $\mathbf{6 9 , 9 2 \%}$ \\
\hline
\end{tabular}

Source: Mičić, 2018

Results achieved: The average wheat yield on Farm B was $7.5 \mathrm{t} /$ ha, ranging from 7.0 $\mathrm{t} /$ ha to $8.0 \mathrm{t} / \mathrm{ha}$.

The realized profit on 3 ha is $2853,00 €$, production economy is 3,33 and profitability of revenue is $69,92 \%$.

Birth weight of lambs in the month of birth showed that most lambs were born in March, with the smallest and highest birth weights, while the smallest lambs were born in April (Table 7).

Table 7. Lamb birth weight, standard deviation, standard average error and deviations in four different months

\begin{tabular}{|l|l|l|l|l|l|l|l|}
\hline $\begin{array}{l}\text { Month of } \\
\text { birth }\end{array}$ & Mean & $\mathbf{N}$ & Std. Deviation & $\begin{array}{l}\text { Std. Error of } \\
\text { Mean }\end{array}$ & Minimum & $\begin{array}{l}\text { Maxi } \\
\text { mum }\end{array}$ & Variance \\
\hline January & 4.88 & 58 & .892 & .118 & 3.12 & 6.58 & .798 \\
\hline March & 4.47 & 238 & .978 & .065 & 2.12 & 6.88 & .955 \\
\hline $\begin{array}{l}\text { Month of } \\
\text { birth }\end{array}$ & Mean & $\mathbf{N}$ & Std. Deviation & $\begin{array}{l}\text { Std. Error of } \\
\text { Mean }\end{array}$ & Minimum & $\begin{array}{l}\text { Maxi } \\
\text { mum }\end{array}$ & Variance \\
\hline April & 4.68 & 25 & .785 & .165 & 3.62 & 6.12 & .615 \\
\hline October & 4.32 & 68 & .942 & .115 & 2.52 & 6.73 & .885 \\
\hline Total & 4.48 & 388 & .963 & .049 & 2.12 & 6.82 & .927 \\
\hline
\end{tabular}

Source: Mičić, 2018

The mean lambs average weight was the highest in January and the lowest average birth weight in October.

The differences for the average birth weight were $0.44 \mathrm{~kg}, 0.21 \mathrm{~kg}$ and $0.58 \mathrm{~kg}$ from January to March, January to April and January to October the month of birth of the lambs.

Analysis of variance showed that birth month had a significant effect on lamb birth weight $(\mathrm{P}<.005)$.

Based on the presented result for 2018, we can conclude that the production of lambs, on farms A and B is economically justified, the financial indicators are given in tabular form, production of lambs and others. on medium ovary farm A and small ovarian farm B in Serbia (Table 8), Mičić et al., (2017). 
Table 8: Economics of production of lambs for sale on medium A and small B sheep farms in Serbia for 2018.

\begin{tabular}{|c|c|c|}
\hline Elements & THE FARM A & THE FARM B \\
\hline A) Number of sheep on the farm & 150 & 75 \\
\hline \multicolumn{3}{|l|}{ PRODUCTION SCOPE } \\
\hline 1. Total volume & 6.750 & 3.375 \\
\hline 2. After a sheep & 45 & 45 \\
\hline \multicolumn{3}{|l|}{ REVENUE } \\
\hline 3. Lambs for sale & 13.500 & 6.750 \\
\hline 4. Milk incentives & 0 & 0 \\
\hline 5. Incentives to die. rejuvenated & 3.000 & 1.500 \\
\hline 6. Sheep wool & 2.250 & 1125 \\
\hline 7. Lambs for overhauling herds (scraps) & 2.250 & 1.125 \\
\hline 6. Wrap milk & 9.000 & 4.500 \\
\hline 9. Manure & 3.000 & 1.500 \\
\hline 10. Sheep hatched & 6.000 & 3.000 \\
\hline B) Total revenue & 38.970 & 19.485 \\
\hline \multicolumn{3}{|l|}{ COSTS } \\
\hline 11. Food & 12.000 & 6.000 \\
\hline 12. Veterinary services and medicines & 750 & 375 \\
\hline 13. Died & 1.500 & 750 \\
\hline 14. Human work & 4.500 & 2.250 \\
\hline 15. Energy and fuel & 1.500 & 750 \\
\hline 16. Depreciation of facilities and equipment & 1.000 & 500 \\
\hline 17. Other & 800 & 400 \\
\hline C) Total cost & 22.050 & 11.025 \\
\hline Elements & THE FARM A & THE FARM B \\
\hline \multicolumn{3}{|l|}{ INCOME / LOSS } \\
\hline D) On the farm & 16.920 & 8.460 \\
\hline 18. Down the throat D:A & 112,80 & 112,79 \\
\hline 19. Economy of production B:C & 1,77 & 1,76 \\
\hline 20. Production Profitability D:Bx 100 & $43,42 \%$ & $43,41 \%$ \\
\hline
\end{tabular}

Source: Mičić, 2018

Based on the result shown, we can conclude that the production of lambs for sale on both farms is economically justified in 2018. (Ripoll et al., 2018).

\section{Conclusions}

Based on the analysis of the situation in our economy, and especially in the livestock breeding industry, specifically lambs for sale, it is necessary to draw some conclusions when it comes to access to this type of production. This primarily refers to the food industry in Serbia. 
This research came to the following conclusions: a more complex analytical review of the conditions and results of development of the aforementioned industry of Serbia in the last two decades, required a preliminary conceptual (re) definition and systematic classification of the activities covered, as well as methodological problems of their informative monitoring.

Two branches of industrial production (food and livestock) based on the processing of primary agricultural products are linked in the supply chain, ie primary production under farm conditions to farms A and B (wheat, maize, barley, triticale and other fodder), farms $\mathrm{A}$ and $\mathrm{B}$ are engaged in the production and production of lambs, and around 700,000 farms bring about $40 \%$ of gross domestic product in Serbia.

The data presented show that the income from the products, plus the incentives, covered the costs of production and gave the rest of the income on the sheep farm A and B.

The total profit realized in the livestock production on farm A for 150 sheep amounts to EUR 22,340.8.

Economy of production 1,77 and profitability of production 43,42\%.

Also on Farm B, the total profit in the livestock production for 75 sheep is EUR 12,624.6.

Economy of production 1.76 and profitability of production $43.41 \%$.

An analysis of variance showed that the month of birth had a significant effect on lamb mass, from the point of view of the influence of the season, the highest body mass was in spring and winter and the lowest in autumn, but despite differences in weight, the season did not show a significant effect on lamb weight $(\mathrm{P}>.005)$.

The highest body weight are have single, then twins and triplets.

\section{Conflict of interests}

The authors declare no conflict of interest.

\section{References}

1. Archimede, H., Pellonde, P., Despois, P., Etienne, T., Alexandre, G. (2008). Growth performances and carcass traits of Ovin Martinik lambs fed various ratios of tropical forage to concentrate under intensive conditions. Small Rumin Res. 75, 162-70.

2. Catalan A., Hernandez A., Fraga L.M., Mireles E.J. (2018). Nongenetic effects on birth weight of MEVEZUG creole sheep in Guerrero, México. Cuban Journal of Agricultural Science, 52, 2,1-9.

3. Gbangboche A.B., Youssao A.K., Senou M., Adamou-Ndlaye M., Ahissou A., Farnir F., Michaux C., Abiola F.A., Leroy P.L (2006). Examination of non-genetic factors affecting the growth performance of djallonke sheep in soudanian zone at the Okpara breeding farm of Benin. Trop Anim Health Prod., 38(1):55-6. 
4. Kegalj Andrijana, Krvavica Marina, Vrdoljak Marija, Ljubičić Iva, Dragaš Marijana, Marulić M. (2011). Current state and trends in production of sheep meat in EU and Croatia, Knin, Croatia.

5. Kukovic, S., Németh, T., Lengyel, A., Toldi, G., Jávor, A. (2013). The Effects Of Genotype And Fattening Technology On Meat Producton Characteristics. Lucrări Ştiințifice-Seria Zootehnie, 59, 11-15.

6. Marina I. Selionova, Galina T. Bobryshova (2017). Sheep breeding economic, pluses and minuses Proceedings of the 11th International Symposium Modern Trends in Livestock Production, October 11-13, 2012, 79-89.

7. Matsushita, M.,Cruz-Martin, Jr A., Marques-Gomes, S.T., de Asis-Fonseca de Macedo, F., Visentainer, J.V., Evalazio de Souza, N. (2010). Influence of slaughter weight on the proximate composition and fatty acid profile of feedlotfattened lamb meat. Acta Scientiarum Technology, 32(3) 315-318.

8. Mellado J., Marin V., Reyes-Carrillo J.L., Mellado M., Gaytan L., De Santiago M. (2016). Effects of non-genetic factors on pre-weaning growth traits in Dorper sheep Managed Intensively In Central Mexico. Ecosistemas y Recursos Agropecuarios, 3(8):229-235.

9. Mičić, I., Rajić, Z., Živković, J., Orović, D., Mičić, M., Mičić, I., I., Mičić, I., M. (2017). Optimal flock structure of pig farm providing minimum costs, Ekonomika poljoprivrede, Vol. 64, No. 3.

10. Michich I., Cvijanovich D., Peshich V. (2018). Economics of Production of Lamb in Serbia, Russian State Agrarian University-MAA named after K.A. Timiryazev Proceedings of the International Scientific and Practical Conference, p. $126-130$. [in Russian: Mičić I., Cvijanović D., Pešić V. (2018). Экономика производства ягнятины в сербии, Россйский государственный аграрный университетМСХА имени К.А. Тимирязева Сборник трудов Международной научнопрактической конференции, p. 126 -130].

11. Momoh O.M., Rotimi E.A., Dim N.I. (2013). Breed effect and non-genetic factors affecting growth performance of sheep in a semi-arid region of Nigeria. Journal of Applied Biosciences, 67:5302 - 5307.

12. Saatchi, M., Miraei-Astiani, S.R., Zare Shahneh, A. (2010). Comparison of Growth and feed-lot traits in Kordi crossbred and purebred lambs (crossbreeding between some Iranian fat-tailed breed) Department of Animal Science Faculty of Agriculture University of Tehran.

13. Rahimi S.M., Rafat S.A., Jafari S. (2014). Effects of environmental factors on growth traits in Makuie sheep. Biotechnology in Animal Husbandry 30 (2), 185192.

14. Ripoll G., Joy Margalida, Panea B. (2018). Consumer Perception of the Quality of Lamb and Lamb Confit, Journal Foods. no. 7. 
15. Siddalingamurthy. H. K ., Manju, G.U., Roopa Devi. Y.S., Manjunatha. S. S., Sreesujatha. R. M. (2017). Non-genetic factors affecting birth and weaning weight in Mandya sheep. Int. J. Adv. Res., 5(4), 345348.

16. Simeonov M.S., Harmon D., Nedelkov K.V. (2015). Non-genetic factors affecting birth weight in the lambs of Blackheads Pleven breed. J. Anim. Sci. Adv., 5(3): 1208-1217.

17. Tohidi R., Javanmard A., Shamsabadi V. (2016). Analysis of the nongenetic factors affecting the growth traits of Balouchi sheep. J. Bio. Env. Sci., 8, 6, 67-73.

18. Zapletal, D., Kuchtik, J., Dobes, I. (2010). The effect of genotype on the chemical and fatty acid composition of the Quadriceps femoris muscle in extensively fattened lambs. Archiev Tierzucht, 5, 589-599.

19. Zaharia, R., Pascal, C., Zaharia, N., Albu, A., Radu-Rusu, R.M. (2013). The Influence Of The Growth System On Dressing Percentage And The Meat Quality Obtained From Turcana Lambs Bred In Montain Area In North-East Of Romania. Lucrări Ştiințifice-Seria Zootehnie, 59,193-198.

20. Yusup A.Yuldashbaev, Maria I. Dongak, Ksenia A. Kulikova, Elena V. Pakhomova, Zhaziraim M. Abenova, Salbak O. Chylbakool, Milan P. Petrovic (2017). The modern state of sheep breeding in russia, Proceedings of the 11th International Symposium Modern Trends in Livestock Production October 11-13, 201, p. 71- 78. 